\section{Rede regionalizada de atenção à saúde no Estado de Santa Catarina, Brasil, entre 2011 e 2015: sistema de governança e a atenção à saúde bucal}

\author{
The regionalized healthcare network in Santa \\ Catarina State, Brazil, from 2011 to 2015: \\ governance system and oral healthcare
}

\section{Red regionalizada de atención a la salud en el Estado de Santa Catarina, Brasil, entre 2011 y 2015: sistema de gobernanza y atención a la salud bucal}

Heloisa Godoi 1

Selma Regina de Andrade 1

Ana Lúcia Schaefer Ferreira de Mello 1

\section{Resumo}

Objetivou-se descrever o sistema de governança empreendido na estruturação da rede regionalizada de atenção à saúde no Estado de Santa Catarina, Brasil, baseando-se na Comissão Intergestores Bipartite (CIB), tendo como foco a estruturação da atenção à saúde bucal. Trata-se de um estudo de cunho qualitativo, exploratório-descritivo, do tipo documental, tendo por base os fundamentos da governança como ferramenta analítica, por meio da identificação das dimensões atores, normas, pontos nodais e processos. Dados secundários foram coletados em atas de reuniões realizadas pela CIB entre janeiro de 2011 $e$ dezembro de 2015. A análise mostra fragilidades na governança empreendida pela CIB catarinense no que concerne à estruturação da atenção à saúde bucal de forma regionalizada e sob a perspectiva de rede. A estruturação da atenção à saúde bucal ocorre de forma paralela a outras redes temáticas no estado e apresenta a expansão dos serviços odontológicos, principalmente de média complexidade, como efeito do processo de governança vigente. As relações estabelecidas entre os gestores e os processos decisórios identificados permitiram reconhecer a "prescrição" dessa rede, visto que há pouca negociação e demanda local, sendo mais restrita ao seguimento de recomendações e incentivos da esfera federal/estadual, estes intermediados por técnicos da secretaria estadual. Dessa forma, a definição de agenda propícia à formulação de política voltada para a atenção à saúde bucal dos catarinenses torna-se fragilizada e apresenta um posicionamento periférico frente às demais ações de saúde.

Administração de Serviços de Saúde; Regionalização; Assistência à Saúde; Saúde Bucal

\section{Correspondência}

H. Godoi

Universidade Federal de Santa Catarina.

Campus Universitário Trindade, Florianópolis, SC 88040-900, Brasil.

heloisagodoi@gmail.com

1 Universidade Federal de Santa Catarina, Florianópolis, Brasil. 


\section{Introdução}

A governança pode ser reconhecida como um elemento que tem função central para a estruturação dos sistemas de saúde. Como processo de ação coletiva, organiza as dinâmicas entre os atores e as normas sociais, com as quais uma sociedade determina a sua conduta, toma e executa suas decisões a fim de alcançar um bem coletivo comum 1,2,3. No contexto atual dos sistemas de saúde, esse processo engloba várias instituições, em diversos níveis, e extrapola os espaços institucionais do Estado ao incluir membros da sociedade civil na tomada de decisão 1,4,5. Dessa forma, a governança assume características complexas, por envolver sujeitos com diferentes interesses e graus de autonomia 6 .

Apesar de existirem estudos 1,2,4,7 sobre o tema governança, em diferentes áreas e sob distintas perspectivas, o conceito continua cercado de imprecisões, sendo utilizado, principalmente no setor saúde, de forma majoritariamente normativa, ou seja, apresentando uma prescrição de princípios e valores 4 .

No entanto, mais do que um modelo institucional normativo e recomendável para as políticas de saúde, o sistema de governança pode ser empregado de distintas maneiras, visto que cada sociedade desenvolve seus sistemas de tomada de decisão, suas interações, resoluções de conflito, bem como suas regras e instituições 4,7,8. O uso analítico da governança permite que interações entre os atores, processos e regras do jogo sejam descritos e explicados, de modo a obter maior compreensão das condutas e de tomada de decisão em determinado sistema de saúde 9 .

A organização do sistema de saúde brasileiro em redes regionalizadas e integradas de atenção, como forma de superar a fragmentação e proporcionar maior integração entre as ações e os serviços de atenção à saúde, em diferentes níveis de complexidade 10 , requer um sistema de governança participativo. Tal modelo configura a oportunidade de abrir espaço para novas formas de interação entre Estado e sociedade, e aliar a descentralização dos serviços à necessidade de integração solidária entre os municípios 9 .

Dentre as redes temáticas empreendidas no âmbito do SUS, a organização da atenção à saúde bucal ganha destaque ao considerar os impactos positivos estabelecidos após a instituição da Política Nacional de Saúde Bucal (PNSB) 11. Com ela, houve ampliação do acesso a partir da reestruturação da atenção básica e da oferta de serviços especializados em saúde bucal 12,13. No entanto, persiste o desafio de integrar os serviços de atenção à saúde bucal em perspectiva regionalizada, atuando sob a ótica das redes de atenção à saúde e cumprindo com o princípio de integralidade do sistema público de saúde 12,13,14.

A adoção de práticas de governança, capazes de garantir a integração dos serviços de saúde e das ações com base nas necessidades de saúde da população, constitui um objeto de pesquisa para sistemas de saúde que têm, em sua orientação epistemológica, a proposta de atuação integrada e regionalizada, como processo organizativo da atenção à saúde, e como forma de garantir sustentabilidade, justiça social, eficiência e efetividade às suas intervenções $1,9,15$.

Em vista da recente operacionalização da atenção à saúde bucal no modelo de rede regionalizada de atenção no país, é possível afirmar que há uma incipiente aplicação da concepção da governança em análises sobre a sua estruturação. Estudos seguindo esse fundamento são necessários para conhecer a conduta empreendida na tomada de decisão e a atuação dos diferentes atores responsáveis pelo desenvolvimento da política e implantação dessa rede, assim como contribuir para os arranjos de governança aprimorados 16 .

Nesse contexto, objetivou-se descrever o sistema de governança empreendido na estruturação da rede regionalizada de atenção à saúde no Estado de Santa Catarina, Brasil, no período de 2011 a 2015 , tendo como foco a estruturação da atenção à saúde bucal e tomando como base o Marco Analítico da Governança proposto por Hufty et al. 4 e Hufty 7,8 .

O estudo sobre a estruturação da atenção à saúde bucal sob a perspectiva de rede regionalizada justifica-se devido tratar-se de âmbito gerencial, sujeito a um processo de governança, no qual diferentes protagonistas desta rede, em diversos níveis, apresentam agendas distintas e desejam que estas sejam atendidas 7 . 


\section{Método}

\section{Desenho do estudo}

Trata-se de um estudo de abordagem qualitativa, de natureza exploratório-descritiva, do tipo documental, realizado baseando-se nos registros das reuniões ordinárias da Comissão Intergestores Bipartite (CIB) do Estado de Santa Catarina, desde janeiro de 2011 a dezembro de 2015.

Pesquisas documentais permitem investigar determinada problemática de forma indireta, por meio do estudo de registros produzidos pelo ser humano, e partem de um amplo e complexo conjunto de dados com o intuito de gerar elementos manipuláveis, estabelecer relações e realizar conclusões 17 .

Como fundamentação teórica utilizou-se o Marco Analítico de Governança, proposto por Hufty et al. 4 e Hufty 7,8 , que visa a reconhecer os elementos que constituem os processos sociais 4 associados à política em estudo; na presente pesquisa, relacionada à estruturação da atenção à saúde bucal no estado catarinense.

\section{Local do estudo}

O trabalho foi realizado baseando-se na instância de negociação intergovernamental do Estado de Santa Catarina (CIB), a qual representa um importante colegiado em nível estadual, sendo responsável por orientar/induzir o processo de regionalização e a constituição das Comissões Intergestores Regionais (CIR), bem como operacionalizar, articular e negociar com os municípios a estruturação da atenção à saúde da população do estado.

A CIB catarinense é composta por 18 membros titulares e respectivos suplentes, conforme regimento, paritariamente distribuídos em nove representantes da Secretaria de Estado da Saúde de Santa Catarina (SES-SC) e nove de secretarias municipais de saúde, os quais são indicados pelo Conselho de Secretários Municipais de Saúde (COSEMS), considerando-se a diversidade intraestatal. As reuniões são realizadas mensalmente e necessitam ao menos de cinco membros representantes do estado e quatro dos municípios. No período em estudo, a participação dos representantes dos municípios ocorreu de forma significativa, com presença de, em média, 10 secretários municipais de saúde, considerados atores estratégicos para este trabalho. Os gestores técnicos, tidos como atores relevantes, apresentam oito representantes nessa comissão e estiveram presentes em coro mínimo (quatro) em ao menos cinco ocasiões.

Esse colegiado pode ser identificado como um espaço nodal formal no processo de governança do sistema de saúde brasileiro, em que convergem atores, processos, tomada de decisão, acordos e elaboração de normas sociais, que apresentam capacidade de influenciar positiva ou negativamente a construção de políticas de saúde. Portanto, constitui um ambiente de interesse para investigação e análise de suas mudanças e dos efeitos que promovem ao desenvolvimento político, ao modelo de gestão 4,7 e à estruturação da atenção à saúde bucal, foco deste estudo.

\section{Coleta dos dados}

No presente trabalho, tomou-se a estruturação da atenção à saúde bucal na perspectiva de rede regionalizada e integrada no Estado de Santa Catarina como o problema a ser investigado no teor das discussões realizadas na CIB, com o intuito de analisar a influência do sistema de governança vigente sobre esta rede temática, entre os meses de janeiro de 2011 e dezembro de 2015 - período no qual as diretrizes para organização das redes de atenção à saúde eram estabelecidas e postas em prática 18 , bem como lançava-se novo instrumento de governança por meio do Decreto no 7.508/2011 19 .

Além das atas, todos os documentos citados e pertinentes à estruturação da atenção à saúde bucal foram consultados para melhor embasamento teórico e análise dos dados, visto que eram documentos tidos como base ou formulados em função do assunto tratado durante as reuniões e em consonância com o objeto em investigação. Tanto as atas quanto os documentos adjacentes são registros públicos e estão disponíveis na página de Internet da SES-SC. 
Para a organização do banco de dados documentais, adotou-se como critério de inclusão as atas que apresentavam pontos de pauta que atendiam o problema em estudo. A Figura 1 apresenta o fluxograma da seleção dos documentos.

\section{Análise dos dados}

Após levantamento e análise preliminar dos documentos, procedeu-se à leitura exaustiva das atas e análise dos dados conforme os preceitos da pesquisa documental 17, em duas etapas: sistematização dos dados documentais e identificação das categorias de análise propostas por Hufty et al. 4 e Hufty 7,8.

$\mathrm{Na}$ primeira etapa, após ordenamento dos 85 pontos de pauta, realizou-se a concepção de 17 códigos, relacionando-os ao tema principal, os quais foram agrupados por similaridade segundo as dimensões analíticas definidas como: cofinanciamento, planejamento e gestão, rede de cuidados ao portador de deficiência, organização da atenção básica, organização da atenção secundária, organização da atenção terciária e organização das estruturas de apoio. Essas dimensões caracterizam os movimentos empreendidos durante o período em estudo e contribuem para delinear os processos referentes à estruturação da rede regionalizada de atenção à saúde bucal em evolução na instância intergestora.

O segundo passo foi relacionado à dissociação dos elementos constitutivos, conforme preceitos do Marco Analítico de Governança 4,7,8, por meio dos quais descrevem-se e explicam-se as interações entre os atores, processos e regras do jogo. Esse Marco permite compreender as condutas e tomada de decisões instituídas em determinada instância, formal ou informal, com base na identificação e análise das seguintes categorias 4,7,8:

\section{Figura 1}

Fluxograma da seleção dos documentos. Florianópolis, Santa Catarina, Brasil, 2016.

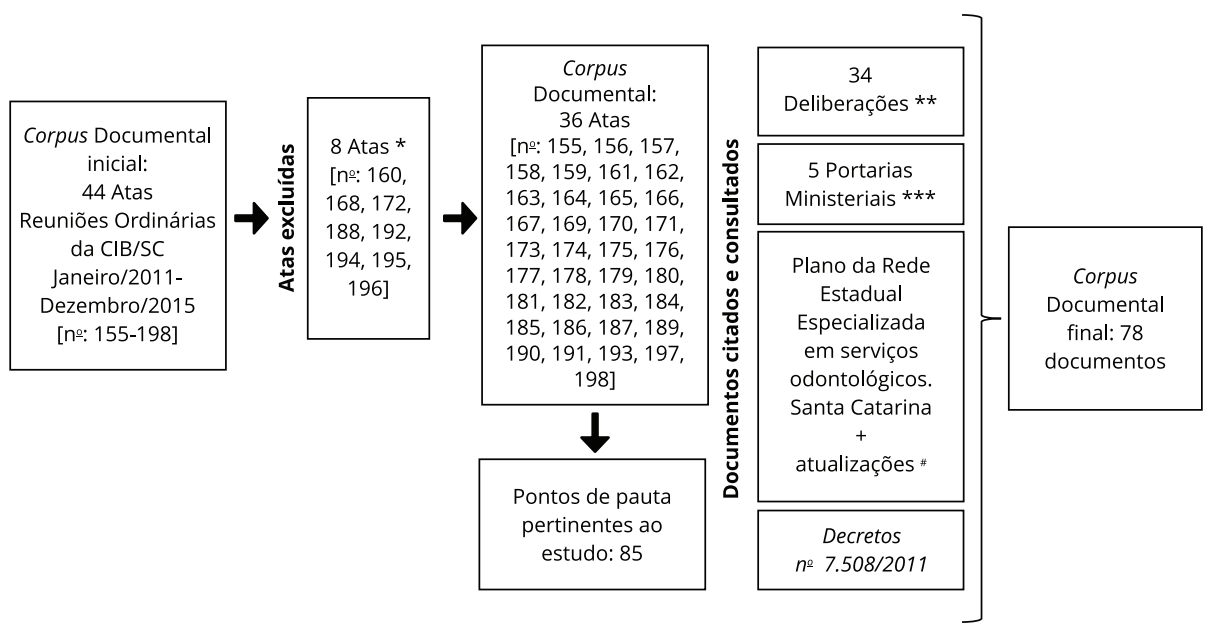

CIB: Comissão Intergestores Bipartite.

* Atas que não atendiam o problema em estudo;

** Deliberações CIB/Santa Catarina: 54/2011; 349/2011; 350/2011; 370/2011; 371/2011; 372/2011; 373/2011; 42/2012;

71/2012; 94/2012; 272/2012; 299/2012; 300/2012; 344/2012; 372/2012; 457/2012; 476/2012; 526/2012; 527/2012;

98/2013; 272/2013; 500/2013; 520/2013; 583/2013; 598/2013; 465/2014; 466/2014; 467/2014; 491/2014; 492/2014;

494/2014; 141/2015; 246/2015;

*** Portarias do Ministério da Saúde: no 2.371/2009; no 1.032/2010; no 4.279/2010; no 2.183/2013; e Portaria

Interministerial no 1.646/2014;

\# Deliberações CIB/Santa Catarina: 77/2005; 222/2007. 
a) Atores: indivíduos envolvidos na trama institucional e na tomada de decisão, podendo ser diferenciados entre estratégicos ou relevantes de acordo com a sua capacidade de influenciar o processo de governança. Neste estudo, foram considerados atores estratégicos os gestores políticos, dentre eles os secretários de saúde; e como relevantes os gestores técnicos, ou seja, coordenadores de distintas áreas na SES-SC. Além disso, a natureza das relações entre os atores também foi estabelecida e pode ser caracterizada como negociação, direção, acordo ou reciprocidade. Na presente pesquisa, realizada com base em dados secundários, as interações dominantes em pauta foram consideradas como Negociação quando havia registro de argumentação de diferentes atores; Direção, no momento em que apenas um ator conduzia/orientava os demais; e Acordo, sempre que havia concordância entre eles; b) Normas: "regras do jogo" ou decisões que orientam o comportamento dos atores, bem como são modificadas pela ação coletiva destes. Podem ser identificadas como meta-normas, normas constitutivas e regulatórias. Os níveis nos quais são empregadas e/ou constituídas também são elementos em estudo.

c) Pontos ou cadeias nodais: espaços físicos ou virtuais dos quais convergem, em um processo de governança, vários problemas, apresentam interação entre atores e normas, em que se realizam tomadas de decisões, concluem acordos e elaboram normas sociais. Podem ser representados pela cadeia formada por outros pontos que fazem parte da trama dos espaços decisórios.

d) Processos: são sucessões de estados pelos quais passam um determinado sistema. Possibilitam a identificação da evolução, ou retrocesso, de um sistema dinâmico, permitindo avaliar a direção para qual evoluem e localizar fatores favoráveis à mudança.

\section{Resultados}

A análise dos dados permitiu identificar dois processos, que estão direta e indiretamente ligados à estruturação da atenção à saúde bucal, no teor dos registros da CIB. Esses processos apresentam dimensões e códigos que determinam a dinâmica e os padrões de evolução da rede, como elementos de uma cadeia explicativa (Tabela 1).

O primeiro processo está associado à Organização da Rede de Atenção à Saúde no Estado de Santa Catarina e está relacionado indiretamente à estruturação da rede regionalizada e integrada de atenção à saúde bucal, sendo constituído pelas dimensões cofinanciamento, planejamento e gestão e rede de cuidados ao portador de deficiência. Essas dimensões atuam de forma transversal no sistema de saúde e demonstram interfaces com a rede de atenção à saúde bucal, por definir estratégias para o financiamento e gestão, bem como a estruturação da rede de cuidados ao portador de deficiência.

Dentre as dimensões encontradas nesse processo, destaca-se o enfoque dado às questões referentes ao financiamento da atenção à saúde em relação aos recursos provenientes do estado, que se apresentam com grande frequência. No entanto, o direcionamento dos recursos para os serviços de atenção básica, determinados pelo gestor estadual e previamente acordados nesta esfera, são prevalentes em comparação aos registros de negociação. A interação predominante no relacionamento entre gestores das duas esferas e técnicos da CIB, referentes a esse assunto, varia entre o estabelecimento de acordos e os direcionamentos realizados pelos representantes do estado sobre a parcela atribuída a este para financiamento das ações e serviços de saúde estabelecidos na atenção básica. Durante o período em estudo, ciclos parecem se formar, alternando entre disponibilidade de valores, suspensão, atrasos e tentativas de negociação que acabam por acordar a proposta previamente estabelecida pelo órgão estadual.

Outro delineamento que ganha destaque, relacionado ao Planejamento e Gestão, diz respeito à implantação de ações pertinentes ao Decreto no 7.508/2011 19, sobre organização, planejamento, assistência à saúde e articulação interfederativa. A CIB, a partir das definições constantes em norma nacional e conduzidas por técnicos da SES-SC, estabelece discussões e acordos, iniciando pela definição de grupo condutor para a implantação do decreto federal, seguida por orientações e solicitações, aos representantes dos municípios, de maior agilidade para formulação e envio dos instrumentos de governança - como Relatório Anual de Gestão e Plano de Saúde, os quais devem ter anuência dos Conselhos de Saúde. A nova conformação das regiões de saúde, também em pauta, é marcada por negociações entre todos os atores atuantes na comissão. 
Tabela 1

Processos de governança relacionados à estruturação da atenção à saúde bucal na Comissão Intergestores Bipartite do Estado de Santa Catarina, Brasil, 2011-2015.

\begin{tabular}{|c|c|c|c|c|c|}
\hline Processo & Dimensão & Código & $\begin{array}{l}\text { Ator } \\
\text { protagonista }\end{array}$ & $\begin{array}{l}\text { Interação } \\
\text { predominante }\end{array}$ & $\begin{array}{l}\text { Pontos de } \\
\text { pauta }\end{array}$ \\
\hline Organização da & Cofinanciamento & Recursos para atenção básica e CEO & Estratégico & Direção & 16 \\
\hline Rede de Atenção & Planejamento e & Ações referentes ao Decreto no 7.508/2011 & Relevante & Direção & 17 \\
\hline \multirow[t]{3}{*}{ à Saúde } & gestão & $\begin{array}{c}\text { Ações referentes à Política Estadual de } \\
\text { Regulação }\end{array}$ & $\begin{array}{l}\text { Estratégico/ } \\
\text { Relevante }\end{array}$ & $\begin{array}{l}\text { Negociação/ } \\
\text { Direção }\end{array}$ & 2 \\
\hline & & $\begin{array}{l}\text { Grupo Condutor para condução de RAS } \\
\text { instituídas pelo Ministério da Saúde }\end{array}$ & Relevante & Acordo & 3 \\
\hline & $\begin{array}{l}\text { Rede de cuidados } \\
\text { ao portador de } \\
\text { deficiência }\end{array}$ & $\begin{array}{c}\text { Adesão de CEO à Rede de cuidados ao } \\
\text { portador de deficiência }\end{array}$ & Relevante & Acordo & 9 \\
\hline $\begin{array}{l}\text { Organização da } \\
\text { Rede de Atenção }\end{array}$ & $\begin{array}{l}\text { Organização da } \\
\text { atenção básica }\end{array}$ & $\begin{array}{l}\text { Recursos para equipamentos odontológicos } \\
\text { atenção básica }\end{array}$ & Estratégico & Acordo & 3 \\
\hline \multirow[t]{11}{*}{ à Saúde Bucal } & & Adesão ao Brasil Sorridente & Estratégico & Acordo & 2 \\
\hline & & Unidade móvel & Relevante & Acordo & 5 \\
\hline & & PET Saúde/GraduaSUS & Relevante & Acordo & 1 \\
\hline & Organização & Implantação de CEO & Relevante & Acordo & 9 \\
\hline & da atenção & Incentivos e ampliação de CEO existente & Relevante & Acordo & 7 \\
\hline & secundária & $\begin{array}{l}\text { Organização de financiamento e referência } \\
\text { para atenção secundária }\end{array}$ & Relevante & Acordo & 3 \\
\hline & & GraduaCEO & Relevante & Acordo & 2 \\
\hline & $\begin{array}{l}\text { Organização da } \\
\text { atenção terciária }\end{array}$ & $\begin{array}{l}\text { Orientação sobre atendimento } \\
\text { odontológico hospitalar }\end{array}$ & Relevante & Direção & 1 \\
\hline & & $\begin{array}{l}\text { Ampliação da especialidade } \\
\text { bucomaxilofacial }\end{array}$ & Estratégico & Negociação & 1 \\
\hline & Organização das & Instituição de LRPD & Estratégico & Acordo & 3 \\
\hline & $\begin{array}{l}\text { estruturas de } \\
\text { apoio }\end{array}$ & Descredenciamento de LRPD & Estratégico & Direção & 1 \\
\hline
\end{tabular}

CEO: Centro de Especialidades Odontológicas; LRPD: Laboratório Regional de Prótese Dentária; PET Saúde: Programa de Educação pelo Trabalho para a Saúde; RAS: Redes de Atenção à Saúde.

No entanto, a preocupação dos gestores municipais quanto à assinatura do Contrato Organizativo de Ação Pública (COAP), presente em todos os anos investigados, sobressai quando comparada aos demais pontos discutidos em pauta. Negociações foram realizadas com o intuito de determinar o melhor momento para a assinatura do termo de compromisso e para que este fosse realizado de forma coerente e responsável, ou seja, contemplando, em mapas de saúde, as reais necessidades e os vazios assistenciais, posto que as diretrizes apontavam como primeira Relação Nacional de Ações e Serviços de Saúde (RENASES) o que estava sendo praticado à data do decreto. Essa conduta, de acordo com as discussões entre gestores registradas em atas, não atendia às reais necessidades da população, como também impunha insegurança financeira e jurídica ao assinarem contrato entre gestores das três esferas de gestão, porém sem representação federal na esfera política.

Além disso, identificou-se a construção da Rede de Cuidado ao Portador de Deficiência e a concepção da Política Estadual de Regulação. Ambas têm interfaces com todas as ações e serviços de saúde. A primeira, porém, está ligada à atenção à saúde bucal, por apresentar subsídios aos Centros de Especialidades Odontológicas (CEO), que realizam adesão à rede de cuidados, como determinado por norma federal, acordado pelos gestores da CIB e seguido por grande parte dos serviços especializados do estado no período em questão. Entretanto, a segunda, apesar de definir centrais de regulação 
macrorregionais para consultas e exames, apresentou maior foco em discussões relacionadas a outros serviços de alta complexidade, os quais são controlados pelas centrais de regulação macrorregional de internações hospitalares e de urgências, sob gestão estadual e fortemente ligada à Rede de Urgência e Emergência (RUE). A RUE, em conjunto com as demais redes temáticas instituídas por portaria ministerial, como rede cegonha e saúde mental, recebeu a constituição de grupos condutores no âmbito da CIB para mobilizar a sua implantação.

Mesmo não apresentando grupo condutor para apoiar/discutir a estruturação da rede regionalizada de atenção à saúde bucal, sua ordenação esteve presente nas pautas da CIB por intermédio do processo identificado como organização da Rede de Atenção à Saúde Bucal, representado pelas dimensões organização da atenção básica, organização da atenção secundária, organização da atenção terciária e organização das estruturas de apoio. A construção desse processo é vista por meio do traçado de implantação dos serviços, a partir de certa prescrição ministerial de serviços de atenção à saúde bucal, principalmente no nível de complexidade secundária e, por conseguinte, primária, sem que grandes discussões ou negociações fossem apresentadas nos documentos analisados. Destaca-se o enfoque dado à ampliação dos serviços ofertados pelos CEO, no período de 2011 a 2015. Nesse período, houve maior incentivo aos serviços regionalizados após a definição, em 2011, de deliberação proposta pelo gestor técnico de saúde bucal da secretaria de estado e aprovada pela CIB, definindo maior custeio estadual destes serviços com base no cofinanciamento da atenção básica. A ampliação do número de CEO no estado, além de seguir a recomendação ministerial para o período, estava fundamentada no Plano da Rede Estadual Especializada em Serviços Odontológicos, elaborado pela comissão técnica de gestores estaduais em 2004, que desenvolveu diagnóstico à época e realizou duas atualizações nos anos 2005 e 2007.

Além da instituição dos serviços, inserções menores relacionadas à integração/interação entre os serviços especializados e os demais serviços da rede foram realizadas, como demandas de dois municípios que solicitaram a alteração da gestão e pactuação. Na estruturação de serviços de atenção básica, além das discussões por recursos do cofinanciamento, o que engloba a conformação de equipes de saúde bucal no primeiro nível de atenção, ocorreu a solicitação ou emissão de unidades odontológicas móveis conforme a Portaria Ministerial no 2.371/2009 e com os recursos provenientes desta. Em relação aos serviços de maior complexidade e às estruturas de apoio, como laboratório regional de prótese dentária, as inserções são tênues e carecem de maior abordagem e discussão.

\section{Discussão}

No presente estudo, adotando-se o enfoque analítico sobre a governança empreendida na rede regionalizada de atenção, identifica-se o desenvolvimento progressivo de ações que têm influência sobre a constituição dos serviços de saúde bucal em Santa Catarina. Tal desenvolvimento é caracterizado pela forte presença de atores ligados ao estado, na determinação de condutas frente aos assuntos pertinentes a toda rede de atenção à saúde, como também aos assuntos direcionados para a estruturação da atenção à saúde bucal. Mesmo em questões referentes ao financiamento, planejamento e gestão, as quais apresentam as maiores discussões e negociações baseando-se nos representantes dos municípios, os atores ligados à esfera estadual tomam protagonismo e direcionam as ações.

No período pesquisado, as deliberações e acordos referentes à rede regionalizada de atenção à saúde bucal ressaltam-se pela concordância estabelecida por técnicos e gestores da esfera estadual, por vezes traduzindo normas ou decretos federais, em contraste às demais redes temáticas que foram estabelecidas pelo Ministério da Saúde e amplamente discutidas nos documentos em questão para que se conformassem de acordo com as necessidades reais da população do estado 6 . Condutas pautadas por uma agenda federal e instituídas a partir do recebimento de recursos federais também são apontadas em outros estudos 9,20 .

O efeito desse processo de governança 4,7,8 sobre a estruturação da rede regionalizada e integrada de atenção à saúde bucal concerne à expansão dos serviços de saúde bucal, principalmente de média complexidade, estabelecendo um processo de organização destes serviços. Apesar de não haver grande negociação sobre integração dos serviços de saúde bucal entre os gestores e discussões sobre demandas apresentadas por estes (provenientes de seus municípios ou a partir da região que estavam 
representando), há que se destacar a preocupação dos técnicos estaduais em estimular a regionalização dos serviços mais complexos por meio de incentivo financeiro estadual, respeitando os princípios de escala e escopo ${ }^{6}$, e sendo influenciada pelo encadeamento de ações estabelecidas para a organização das Regiões de Saúde no Estado e suas respectivas CIR.

Questões sobre o financiamento do setor saúde, apontado como dimensão fundamental para governança das redes 6, são objeto de preocupação e discussão em diversos contextos 9,21 e apresentam ainda mais inquietudes no atual momento brasileiro 22. Em recente pesquisa 20, na qual os autores analisaram a dinâmica de funcionamento da CIB em 24 estados brasileiros, incluindo Santa Catarina, constatou-se que os debates sobre financiamento são bastante frequentes e evidenciam-se por relações de conflito entre seus entes. Assim como houve grande destaque às negociações relativas à consolidação do processo de regionalização, conforme objetivos do Decreto no 7.508/2011. Tal discussão também foi evidenciada no presente estudo.

A CIB catarinense pode ser classificada como avançada 20 quanto à sua institucionalidade (solidez e maturidade quanto espaço de negociação), com discussões diversificadas, aderentes à realidade estadual, tendo agenda compartilhada entre os governos municipais e estadual com base em um relacionamento cooperativo e interativo, e com elevada capacidade de elaborar e implementar políticas. No entanto, no período analisado neste trabalho, os achados corroboram em parte as evidências trazidas por esses autores, visto que se reconhece a legitimidade e organização da CIB como espaço de negociação em Santa Catarina, bem como a sua elevada capacidade de atuação. Porém, no que tange aos aspectos relacionados diretamente à estruturação da atenção à saúde bucal, na perspectiva de rede regionalizada de atenção à saúde, os debates/negociações são substituídos por acordos ou direcionamentos protagonizados por técnicos da esfera estadual. Esses técnicos possuem articulação limitada ao cumprimento/adequação de normas federais, por vezes restritas à aprovação de pleitos e adesões a estratégias ministeriais específicas e condicionadas ao financiamento federal ou estadual.

A ausência de agenda para discutir a concepção de uma política de saúde bucal para o estado catarinense, com base na construção de rede regionalizada de atenção à saúde bucal, com participação e discussão dos atores estratégicos instituídos em instância estadual e responsáveis pela definição de políticas e operacionalização destas, poderia ser comparada à falta de agenda própria, limitada por pautas induzidas por política nacional, identificada por Machado et al. 20 em determinados estados e em região de saúde estudada por Santos et al. ${ }^{9}$. Dessa forma, a definição de agenda propícia à formulação de política voltada para a atenção à saúde bucal dos catarinenses torna-se fragilizada e apresenta, nesta instância, processos de governança com pautas induzidas por demanda ministerial/estadual em detrimento de um planejamento com base em questões locorregionais 14,23, ou seja, construído por gestores estratégicos e fundamentado em discussões prévias facilitadas por um grupo condutor, conforme observado para o estabelecimento de outras redes temáticas no estado.

No entanto, a influência de condicionantes histórico-estruturais pode contribuir para o posicionamento periférico ocupado pela atenção à saúde bucal na instituição de políticas públicas não apenas no estado catarinense, mas também em outros contextos. Tanto no Brasil, mesmo após grandes avanços 13 , como em outros países 24,25 , a atenção à saúde bucal encontra-se marginalmente incluída nas discussões e, por vezes, apresenta-se descolada dos assuntos referentes à organização da atenção à saúde na perspectiva de rede 23 . Ademais, o ensino odontológico continua a priorizar a formação para a atuação no setor privado 13,21 .

Dessa maneira, para que a atenção à saúde bucal possa ser incorporada à atenção à saúde e instituída como rede temática - tendo condição específica por meio de um ciclo completo de atendimento e ações 26 e, portanto, suscetível a um processo de governança 6 - algumas limitações precisam ser superadas, atingindo assim patamares superiores de implantação de política pública, sendo esta discutida no âmbito estadual.

Dentre as frentes de trabalho para superar essas dificuldades discutidas em outros estudos, estariam: a criação de sistemas de saúde mais integrados e com atuação interdisciplinar 24,27, o qual garanta integralidade do cuidado, qualidade e maior acesso a todos os cidadãos brasileiros 21; o fortalecimento na formação de recursos humanos voltados para uma abordagem coletiva 23,27 e não mais exclusivamente para atender a interesses privados, visto que este mercado atende menos de $40 \%$ da população brasileira 13,21,23; a ampliação do financiamento público nessa área, que enfrenta uma gama de necessidades acumuladas ao longo dos anos, e a crescente demanda de serviços pela 
população 13,21,23; maior mudança no aspecto cultural e social frente ao cuidado à saúde bucal, que ainda é tido como secundário e não percebido como direito social 13,26; assim como a inserção de pautas sobre universalização e integralidade da atenção à saúde bucal na agenda de atores estratégicos locais, regionais e também estaduais que apresentam maior capacidade para influenciar o processo de governança, e a instituição de políticas como forma de adquirir maior representatividade, tanto em espaços de planejamento e gestão como de controle social, a fim de fortalecer a saúde bucal como direito de cidadania 28.

A alocação de recursos financeiros empreendida por governantes para determinadas políticas, ações e serviços de saúde também permite indicar prioridades estipuladas 26. A PNSB e políticas estaduais de saúde bucal podem ser vistas como um exemplo de política claramente condicionada pelo seu aporte de recursos financeiros 26 . Nos processos de governança identificados no presente estudo, observou-se a participação e comprometimento do estado catarinense em manter contínuo os recursos para a atenção básica em saúde e saúde bucal, bem como para os serviços odontológicos especializados, sendo que há distintos estímulos financeiros que podem induzir à regionalização da média complexidade, mesmo havendo impasses e ciclos viciosos que precisam ser superados para melhor e maior disponibilização destes recursos. Esses achados diferenciam dos encontrados por Lorena Sobrinho \& Santo 29 , que não constataram a presença de recursos do estado para o financiamento da atenção à saúde bucal em um município pernambucano de grande porte e, conforme Santos e colaboradores 30 , há visível falta de empenho por parte do estado pernambucano em financiar ações de atenção básica.

Contudo, a constatação da participação do estado catarinense no financiamento dos serviços de atenção básica e de média complexidade odontológicos não revoga a máxima existente de os municípios serem os maiores responsáveis pelos recursos destinados à atenção básica 29,31.

A regionalização dos serviços de saúde e a organização destes em redes de atenção são alvo de frequentes debates nos diversos estados brasileiros 20, visto o papel indutor dos estados, por meio das CIB, na conformação das regiões de saúde, no fortalecimento das CIR, na elaboração dos planejamentos regionais e no processo de contratualização entre os entes federativos, por meio do COAP 19. Assim como em outros estados 32, um grupo condutor, formado por representantes do estado e municípios, foi instituído ao final de 2011 para discutir a implantação do Decreto no $7.508 / 2011$ 19. Discussões relacionadas a esse Decreto permearam as atas durante todo o período em estudo e demonstraram o comprometimento em construir um novo modelo de atenção à saúde, voltado para as necessidades da população e desenvolvido em uma rede interfederativa eficaz e sólida para o SUS 31,32,33, bem como evidenciaram a apreensão compartilhada por outros estados 32,34 na assinatura do contrato interfederativo, que foi efetivado até o ano de 2015, por regiões de saúde estabelecidas em apenas dois estados brasileiros 32,35 . A organização de serviços públicos de saúde por meio de contratos de gestão tem se tornado uma importante pauta nas agendas de discussão de gestores e demais stakeholders em diversos países, e a contratualização de resultados é apontada como uma abordagem que busca por eficiência na gestão dos serviços e na construção de políticas públicas 33,36.

Ademais, a constituição de grupos condutores, ou grupos técnicos de trabalho, pode contribuir com a institucionalidade, legitimidade e capacidade de instituir ou influenciar políticas de saúde da CIB 20 e, deste modo, fortalecer a governança empreendida por esta instância 6 . Além de uma equipe para tratar dos assuntos pertinentes ao Decreto, mais um grupo foi instituído com o intuito de mobilizar agentes políticos nas três esferas de gestão e implantar/expandir as redes de atenção determinadas por norma ministerial 18, ou ainda, as que poderiam vir a ser instituídas por norma estadual.

Embora houvesse a possibilidade de organizar novos grupos para a instituição de novas redes, a atenção à saúde bucal não recebeu tal direcionamento, mesmo tendo elementos que justifiquem esta conformação 13,26, dentre os quais a complexidade assistencial, a transposição por diversos níveis de atenção e a necessidade de reduzir as iniquidades de saúde bucal persistentes entre níveis sociais e regiões 26. A constituição de grupos técnicos regulares pode contribuir para uma maior consistência dos debates e decisões conjuntas acerca das políticas de saúde 20 . Sendo assim, seria capaz de fundamentar maior debate sobre a organização regionalizada da atenção em saúde bucal nas instâncias responsáveis por operacionalizar a saúde no estado, tais como as comissões intergestores em âmbito estadual e regional, as quais estão estabelecidas no Estado de Santa Catarina. 
A expansão dos serviços de saúde bucal, delineada pelo processo Organização da Rede de Atenção à Saúde Bucal e vista como efeito da governança empreendida para a estruturação desta rede, mostra a implantação de alguns pressupostos da PNSB implementada em 2004 11, tais como política de financiamento para o desenvolvimento das ações e ampliação das atenções básica e secundária. No entanto, assim como em outros contextos brasileiros 12,13 e ratificando estudos realizados em Santa Catarina 14,23, a integração entre os diversos níveis de complexidade continua sendo um óbice a ser superado, como também a necessidade em consolidar e ampliar os serviços odontológicos em ambiente hospitalar, de modo a transcender os serviços de traumatologia e garantir a articulação deste aos demais pontos de atenção da rede 14,23. Além dessas barreiras, a ausência de discussões sobre regulação dos serviços de saúde bucal, por meio do complexo regulador instituído no estado, demonstra a baixa integralidade e contribui para limitar o desenvolvimento eficiente dessa rede.

Dessa forma, a pouca discussão empreendida pelos gestores estratégicos (com capacidade de influência), na instância de governança investigada, sobre a conformação de uma rede regionalizada e integrada de atenção à saúde bucal, poderia contribuir para justificar a limitação em estabelecer a integralidade do sistema de saúde, incluindo-se os serviços de saúde bucal e, portanto, demonstrar fragilidades no processo de governança empreendido por essa CIB. O cuidado à saúde bucal tem argumentos suficientes para tomar um maior espaço na agenda de discussões sobre a estruturação das redes regionalizadas de atenção à saúde e, deste modo, poderia apresentar maior representação política nessas instâncias (CIB, CIR) que agregam poder decisório, seja por meio de órgão assessor de gestão 6 ou câmaras técnicas 6,20 e, consequentemente, alcançar outro patamar de atenção e assistência 23 .

O estudo permitiu documentar a problemática frente à estruturação de redes regionalizadas, com foco na atenção à saúde bucal, e descrever processos decisórios empreendidos por meio da articulação dos atores, das normas evidenciadas e dos processos em movimento para o advento da organização da atenção à saúde bucal, destacando o protagonismo de atores relevantes, as relações de concordância e os efeitos da governança identificada apenas na ampliação dos serviços odontológicos.

As limitações deste trabalho são inerentes ao seu próprio desenho, que se distancia dos processos de participação social por ser desenvolvido no âmbito de comissões intergestores, e restringe a percepção das relações entre os atores por se basear nos registros realizados durante as reuniões. Assim, sugere-se que estudos futuros com o propósito de analisar a estruturação da atenção à saúde bucal na perspectiva das redes de atenção, sob o foco da governança, sejam realizados em órgãos que representam maior participação da sociedade civil e maior proximidade às realidades regionais, por meio das CIR, bem como considerem a percepção desses atores sobre a constituição das redes. A descentralização e regionalização das ações e serviços de saúde são princípios organizativos consolidados que visam à integralidade da atenção e maior capacidade de resposta às necessidades locais 1,35 , com base na construção política que incentive diálogos entre atores locais e gestores federados.

\section{Colaboradores}

H. Godoi e A. L. S. F. Mello atuaram na concepção e projeto do artigo, análise e interpretação dos dados, redação e revisão crítica relevante do conteúdo intelectual, bem como da aprovação final da versão a ser publicada. S. R. Andrade participou da redação, revisão crítica do conteúdo intelectual do artigo, bem como da aprovação final da versão a ser publicada. 


\section{Referências}

1. Ciccone DK, Vian T, Maurer L, Bradley EH Linking governance mechanisms to health outcomes: a review of the literature in lowand middle-income countries. Soc Sci Med 2014; 117:86-95.

2. Barbazza E, Telo JE. A review of health governance: definitions, dimensions and tools to govern. Health Policy 2014; 116:1-11.

3. Smith PC, Anell A, Busse R, Crivelli L, Healy J, Lindahl AK, et al. Leadership and governance in seven developed health systems. Health Policy 2012; 106:37-49.

4. Hufty M, Báscolo E, Bazzani R. Gobernanza en salud: un aporte conceptual y analítico para la investigación. Cad Saúde Pública 2006; 22 Suppl:S35-45.

5. Jakubowski E, Saltman RB. The changing national role in health system governance: a casebased study of 11 European countries and Australia. Copenhagen: WHO Regional Office for Europe; 2013.

6. Mendes EV. As redes de atenção à saúde. 2a Ed. Brasília: Organização Pan-Americana da Saúde; 2011.

7. Hufty M. Gobernanza en salud pública: hacia un Marco Analítico. Rev Salud Pública 2010; 12 Suppl:39-61.

8. Hufty M. Investigating policy process: the Governance Analytical Framework (GAF). In: Wiesmann U, Hurni H, editors. Research for sustainable development: foundations, experiences, and perspectives. Bern: Geographica Bernensia; 2011. p. 403-24.

9. Santos AM, Giovanella L. Governança regional: estratégias e disputas para gestão em saúde. Rev Saúde Pública 2014; 48:622-31.

10. Vargas I, Mogollón-Pérez AS, Unger JP, Ferreira-da-Silva MR, Paepe P, Vázquez ML. Regional-based Integrated Healthcare Network policy in Brazil: from formulation to practice. Health Policy Plan 2015; 30:705-17.

11. Coordenação Nacional de Saúde Bucal, Departamento de Atenção Básica, Secretaria de Atenção à Saúde, Ministério da Saúde. Diretrizes da Política Nacional de Saúde Bucal. http://189.28.128.100/dab/docs/publicacoes/ geral/diretrizes_da_politica_nacional_de_sau de_bucal.pdf (acessado em 10/Jul/2016).

12. Aquilante AG, Aciole GG. Construindo um "Brasil Sorridente?". Olhares sobre a implementação da Política Nacional de Saúde Bucal numa região de saúde do interior paulista. Cad Saúde Pública 2015; 31:82-96.

13. Pucca Jr. GA, Gabriel M, Araujo ME, Almeida FCS. Ten years of a National Oral Health Policy in Brazil: innovation, boldness, and numerous challenges. J Dent Res 2015; 94:1333-7.

14. Godoi H, Mello ALSF, Caetano JC. Rede de atenção à saúde bucal: organização em municípios de grande porte de Santa Catarina, Brasil. Cad Saúde Pública 2014; 30:318-32.
15. Nicholson C, Jackson C, Marley J. A governance model for integrated primary/ secondary care for the health-reforming first world - results of a systematic review. BMC Health Serv Res 2013; 13:528.

16. Batchelor P. Improving governance to improve oral health: addressing care delivery systems. Oral Health Dent Manag 2012; 11:129-33.

17. Flick U. Introdução à pesquisa qualitativa. 3a Ed. Porto Alegre: Editora Artmed; 2009.

18. Ministério da Saúde. Portaria no 4.279, de 30 de dezembro de 2010, estabelece diretrizes para a organização da Rede de Atenção à Saúde no âmbito do Sistema Único de Saúde (SUS). Diário Oficial da União 2010; $31 \mathrm{dez}$

19. Presidência da República. Decreto no 7.508, de 28 de junho de 2011. Regulamenta a Lei no 8.080, de 19 de setembro de 1990, para dispor sobre a organização do SUS, o planejamento da saúde, a assistência à saúde e a articulação interfederativa, e dá outras providências. Diário Oficial da União 2011; 29 jun.

20. Machado CV, Lima LD, Viana ALA, Oliveira RG, Iozzi FL, Albuquerque MV, et al. Federalismo e política de saúde: comissões intergovernamentais no Brasil. Rev Saúde Pública 2014; 48:642-50.

21. Nascimento AC, Moyses ST, Werneck RI, Moyses SM. Oral health in the context of primary care in Brazil. Int Dent J 2013; 63:237-43.

22. Santos AO, Delduque MC, Alves SMC. Os três poderes do Estado e o financiamento do SUS: o ano de 2015. Cad Saúde Pública 2016; 32:e00194815.

23. Mello ALSF, Andrade SR, Moyses SJ, Erdmann AL. Saúde bucal na rede de atenção e processo de regionalização. Ciênc Saúde Coletiva 2014; 19:205-14.

24. Sparer M. US health care reform and the future of dentistry. Am J Public Health 2011; 101:1841-4.

25. Schwab GL, Moyses ST, França BHS, Werneck RI, Frank E, Moyses SJ. Chronic conditions policies: oral health, a felt absence. Int Dent J 2014; 64:83-8.

26. Moyses SJ. Saúde coletiva: políticas, epidemiologia da saúde bucal e redes de atenção odontológica. São Paulo: Editora Artes Médicas; 2013.

27. Scherer CI, Scherer MDA. Avanços e desafios da saúde bucal após uma década de Programa Brasil Sorridente. Rev Saúde Pública 2015; 49:98.

28. Alves-Souza RA, Saliba O. A saúde bucal em pauta: análise de registros dos Conselhos $\mathrm{Mu}-$ nicipais de Saúde de municípios pertencentes a 17a Regional de Saúde do Estado do Paraná, Brasil. Cad Saúde Pública 2003; 19:1381-8. 
29. Lorena Sobrinho JE, Santo ACGE. Participação dos entes federados no financiamento da saúde bucal de atenção básica: estudo no $\mathrm{Mu}$ nicípio da Vitória de Santo Antão, Pernambuco. Saúde Soc 2013; 22:994-1000.

30. Santos FAS, Gurgel Jr GD, Pacheco HF, Marteli PJL. A regionalização e financiamento da saúde: um estudo de caso. Cad Saúde Colet (Rio J.) 2015; 23:402-8.

31. Pinafo E, Carvalho BG, Nunes EFPA. Descentralização da gestão: caminho percorrido, nós críticos e perspectivas. Ciênc Saúde Coletiva 2016; 21:1511-24.

32. Tavares FA, Lima MS, Pereira LA, Santos MCP, Nicolato BCS. Novas perspectivas na gestão para resultados na saúde em Minas Gerais: a implantação dos contratos organizativos de ação pública de saúde. In: Anais do VI Congresso CONSAD de Gestão Pública. Brasília: Conselho Nacional de Secretários de Estado de Administração; 2013. p. 16-18.
33. Shimizu HE. Percepção dos gestores do Sistema Único de Saúde acerca dos desafios da formação das Redes de Atenção à Saúde no Brasil. Physis (Rio J.) 2013; 23:1101-22.

34. Mendes A, Louvison MCP, Ianni AMZ, Leite MG, Feuerwerker LCM, Tanaka OY, et al. O processo de construção da gestão regional da saúde no Estado de São Paulo: subsídios para a análise. Saúde Soc 2015; 24:423-37.

35. Moura DCN, Pinto JR, Aragão AEA. Perfil dos profissionais atuantes na gestão em saúde frente ao novo modelo de reorganização do SUS: a regionalização. Tempus (Brasília) 2016; 10:75-93.

36. Ditterich RG, Moyses ST, Moyses SJ. O uso de contratos de gestão e incentivos profissionais no setor público de saúde. Cad Saúde Pública 2012; 28:615-27. 


\section{Abstract}

The objective was to describe the governance system used in structuring the regionalized healthcare network in Santa Catarina State, Brazil, based on the Bipartite Inter-Managerial Commission (CIB), with a focus on structuring of oral healthcare. This was a qualitative, exploratory-descriptive documental study, based on the foundations of governance as an analytical tool through identification of the dimensions actors, norms, nodal points, and processes. Secondary data were collected from the minutes of CIB meetings held from January 2011 to December 2015. The analysis shows weaknesses in CIB governance in Santa Catarina in relation to regionalized structuring of oral healthcare from a network perspective. Structuring of oral healthcare occurs in parallel to that of other thematic networks in the state and shows the expansion of dental services, especially those with medium complexity, as an effect of the prevailing governance process. The relations established between administrators and decision-making processes allowed recognizing this network's "prescription", since there is little negotiation and local demand, limited more to following recommendations and incentives from the federal/state sphere, intermediated by staff from the State Health Secretariat. Thus, setting a policy agenda for oral healthcare for the population of Santa Catarina is weakened, with a peripheral position in relation to other health programs.

Health Services Administration; Regional Health Planning; Delivery of Health Care; Oral Health

\section{Resumen}

El objetivo fue describir el sistema de gobernanza emprendido en la estructuración de la red regionalizada de atención a la salud, en el estado de Santa Catarina, basándose en la Comisión Intergestores Bipartita (CIB), cuyo propósito fue la estructuración de la atención a la salud bucal. Se trata de un estudio de cuño cualitativo, exploratorio-descriptivo, de tipo documental, teniendo como base los fundamentos de la gobernanza, como herramienta analitica, mediante la identificación de las dimensiones: actores, normas, puntos nodales $y$ procesos. Los datos secundarios se recogieron en las actas de reuniones realizadas por la CIB, entre enero de 2011 y diciembre de 2015. El análisis muestra debilidades en la gobernanza emprendida por la CIB catarinense, en lo que concierne a la estructuración de la atención a la salud bucal de forma regionalizada $y$ desde su perspectiva de red. La estructuración de la atención a la salud bucal se produce de forma paralela a otras redes temáticas en el estado y presenta la expansión de servicios odontológicos, principalmente de media complejidad, como consecuencia del proceso de gobernanza vigente. Las relaciones establecidas entre los gestores y los procesos decisorios identificados permitieron reconocer la "prescripción" de esta red, visto que existe poca negociación y demanda local, estando más restringida al seguimiento de recomendaciones e incentivos de la esfera federal/ estatal, además intermediada por técnicos de la secretaría estatal. De esta forma, la definición de esta agenda, propicia a la formulación de políticas dirigidas hacia la atención a la salud bucal de los catarinenses, se debilita y presenta una posición periférica frente a las demás acciones de salud.

Administración de los Servicios de Salud;

Regionalización; Prestación de Atención de Salud;

Salud Bucal
Recebido em 28/Jul/2016

Versão final reapresentada em 09/Nov/2016

Aprovado em 28/Nov/2016 